\title{
Sufficient conditions for starlike functions associated with the lemniscate of Bernoulli
}

\author{
S Sivaprasad Kumar ${ }^{1}$, Virendra Kumar ${ }^{1}$, V Ravichandran ${ }^{2}$ and Nak Eun Cho ${ }^{3 *}$
}

${ }^{*}$ Correspondence:

necho@pknu.ac.kr

${ }^{3}$ Department of Applied

Mathematics, Pukyong National

University, Busan, 608-737, South

Korea

Full list of author information is

available at the end of the article

\begin{abstract}
Let $-1 \leq B<A \leq 1$. The condition on $\beta$ is determined so that

$1+\beta z p^{\prime}(z) / p^{k}(z) \prec(1+A z) /(1+B z)(-1<k \leq 3)$ implies $p(z) \prec \sqrt{1+z}$. Similarly, the condition on $\beta$ is determined so that $1+\beta z p^{\prime}(z) / p^{n}(z)$ or $p(z)+\beta z p^{\prime}(z) / p^{n}(z) \prec \sqrt{1+z}$ $(n=0,1,2)$ implies $p(z) \prec(1+A z) /(1+B z)$ or $\sqrt{1+z}$. In addition to that, the condition on $\beta$ is derived so that $p(z) \prec(1+A z) /(1+B z)$ when $p(z)+\beta z p^{\prime}(z) / p(z) \prec \sqrt{1+z}$. A few more problems of the similar flavor are also considered.
\end{abstract}

MSC: $30 \mathrm{C} 80 ; 30 \mathrm{C} 45$

Keywords: starlike function; lemniscate of Bernoulli; subordination

\section{Introduction}

Let $\mathcal{A}$ be the class of analytic functions defined on the unit disk $\mathbb{D}:=\{z \in \mathbb{C}:|z|<1\}$ normalized by the condition $f(0)=0=f^{\prime}(0)-1$. For two analytic functions $f$ and $g$, we say that $f$ is subordinate to $g$ or $g$ is superordinate to $f$, denoted by $f \prec g$, if there is a Schwarz function $w$ with $|w(z)| \leq|z|$ such that $f(z)=g(w(z))$. If $g$ is univalent, then $f \prec g$ if and only if $f(0)=g(0)$ and $f(\mathbb{D}) \subseteq g(\mathbb{D})$. For an analytic function $\varphi$ whose range is starlike with respect to $\varphi(0)=1$ and is symmetric with respect to the real axis, let $\mathcal{S}^{*}(\varphi)$ denote the class of Ma-Minda starlike functions consisting of all $f \in \mathcal{A}$ satisfying $z f^{\prime}(z) / f(z) \prec \varphi(z)$. For special choices of $\varphi, \mathcal{S}^{*}(\varphi)$ reduces to well-known subclasses of starlike functions. For example, when $-1 \leq B<A \leq 1, \mathcal{S}^{*}[A, B]:=\mathcal{S}^{*}((1+A z) /(1+B z))$ is the class of Janowski starlike functions [1] (see [2]) and $\mathcal{S}^{*}[1-2 \alpha,-1]$ is the class $\mathcal{S}^{*}(\alpha)$ of starlike functions of order $\alpha$ and $\mathcal{S}^{*}:=\mathcal{S}^{*}(0)$ is the class of starlike functions. For $\varphi(z):=\sqrt{1+z}$, the class $\mathcal{S}^{*}(\varphi)$ reduces to the class $\mathcal{S} \mathcal{L}$ introduced by Sokół and Stankiewicz [3] and studied recently by Ali et al. [4,5]. A function $f \in \mathcal{A}$ is in the class $\mathcal{S} \mathcal{L}$ if $z f^{\prime}(z) / f(z)$ lies in the region bounded by the right half-plane of the lemniscate of Bernoulli given by $\left|w^{2}-1\right|<1$. Analytically, $\mathcal{S L}:=\left\{f \in \mathcal{A}:\left|\left(z f^{\prime}(z) / f(z)\right)^{2}-1\right|<1\right\}$. For $b \geq 1 / 2$ and $a \geq 1$, a more general class $S^{*}[a, b]$ of the functions $f$ satisfying $\left|\left(z f^{\prime}(z) / f(z)\right)^{a}-b\right|<b$ was considered by Paprocki and Sokół [6]. Clearly, $S^{*}[2,1]=: \mathcal{S L}$. For some radius problems related with the lemniscate of Bernoulli, see $[3,5,7,8]$. Estimates for the initial coefficients of functions in the class $\mathcal{S} \mathcal{L}$ are available in [8].

Let $p$ be an analytic function defined on $\mathbb{D}$ with $p(0)=1$. Recently Ali et al. [4] determined conditions for $p(z) \prec \sqrt{1+z}$ when $1+\beta z p^{\prime}(z) / p^{k}(z)$ with $k=0,1,2$ or $(1-\beta) p(z)+$ $\beta p^{2}(z)+\beta z p^{\prime}(z)$ is subordinated to $\sqrt{1+z}$. Motivated by the works in [4-8], in Section 2 the condition on $\beta$ is determined so that $p(z) \prec \sqrt{1+z}$ when $1+\beta z p^{\prime}(z) / p^{k}(z) \prec(1+A z) /(1+$ $B z)(-1<k \leq 3)$. Similarly, the condition on $\beta$ is determined so that $p(z) \prec(1+A z) /(1+B z)$

\section{黛 Springer}

(c) 2013 Kumar et al.; licensee Springer. This is an Open Access article distributed under the terms of the Creative Commons Attribution License (http://creativecommons.org/licenses/by/2.0), which permits unrestricted use, distribution, and reproduction in any medium, provided the original work is properly cited. 
when $1+\beta z p^{\prime}(z) / p^{n}(z) \prec \sqrt{1+z}, n=0,1,2$. Further, the condition on $\beta$ is obtained in each case so that $p(z) \prec \sqrt{1+z}$ when $p(z)+\beta z p^{\prime}(z) / p^{n}(z) \prec \sqrt{1+z}, n=0,1,2$. At the end of this section, the problem $p(z)+\beta z p^{\prime}(z) / p(z) \prec \sqrt{1+z}$ implies $p(z) \prec(1+A z) /(1+B z)$ is also considered.

Silverman [9] introduced the class $\mathcal{G}_{b}$ by

$$
\mathcal{G}_{b}:=\left\{f \in \mathcal{A}:\left|\frac{z f^{\prime \prime}(z) / f^{\prime}(z)}{z f^{\prime}(z) / f(z)}-1\right|<b\right\}
$$

and proved $\mathcal{G}_{b} \subset \mathcal{S}^{*}(2 /(1+\sqrt{1+8 b})), 0<b \leq 1$. Further, this result was improved by Obradovič and Tuneski [10] by showing $\mathcal{G}_{b} \subset S^{*}[0, b] \subset \mathcal{S}^{*}(2 /(1+\sqrt{1+8 b})), 0<b \leq 1$. Tuneski [11] further obtained the condition for $\mathcal{G}_{b} \subset S^{*}[A, B]$. Inspired by the work of Silverman [9], Nunokawa et al. [12] obtained the sufficient conditions for a function in the class $\mathcal{G}_{b}$ to be strongly starlike, strongly convex, or starlike in $\mathbb{D}$. By setting $p(z)=$ $z f^{\prime}(z) / f(z)$, the inclusion $\mathcal{G}_{b} \subset S^{*}[A, B]$ can be written as

$$
1+\frac{z p^{\prime}(z)}{p^{2}(z)} \prec 1+b z \quad \Longrightarrow \quad p(z) \prec \frac{1+A z}{1+B z}
$$

Recently Ali et al. [13], obtained the condition on the constants $A, B, D, E \in[-1,1]$ and $\beta$ so that $p(z) \prec(1+A z) /(1+B z)$ when $1+\beta z p^{\prime}(z) / p^{n}(z) \prec(1+D z) /(1+E z), n=0,1$. In Section 3 , alternate and easy proofs of results [13, Lemmas 2.1, 2.10] are discussed. Further, this section is concluded with the condition on $A, B, D, E \in[-1,1]$ and $\beta$ such that $1+$ $\beta z p^{\prime}(z) / p^{2}(z) \prec(1+D z) /(1+E z)$ implies $p(z) \prec(1+A z) /(1+B z)$.

The following results are required in order to prove our main results.

Lemma 1.1 [14, Corollary 3.4h, p.135] Let $q$ be univalent in $\mathbb{D}$, and let $\varphi$ be analytic in a domain D containing $q(\mathbb{D})$. Let $z q^{\prime}(z) \varphi(q(z))$ be starlike. If $p$ is analytic in $\mathbb{D}, p(0)=q(0)$ and satisfies

$$
z p^{\prime}(z) \varphi(p(z)) \prec z q^{\prime}(z) \varphi(q(z))
$$

then $p \prec q$ and $q$ is the best dominant.

The following is a more general form of the above lemma.

Lemma 1.2 [14, Corollary 3.4i, p.134] Let $q$ be univalent in $\mathbb{D}$, and let $\varphi$ and $v$ be analytic in a domain D containing $q(\mathbb{D})$ with $\varphi(w) \neq 0$ when $w \in q(\mathbb{D})$. Set

$$
Q(z):=z q^{\prime}(z) \varphi(q(z)), \quad h(z):=v(q(z))+Q(z)
$$

\section{Suppose that}

(1) $h$ is convex or $Q(z)$ is starlike univalent in $\mathbb{D}$ and

(2) $\operatorname{Re}\left(\frac{z h^{\prime}(z)}{Q(z)}\right)>0$ for $z \in \mathbb{D}$.

If

$$
v(p(z))+z p^{\prime}(z) \varphi(p(z)) \prec v(q(z))+z q^{\prime}(z) \varphi(q(z)),
$$

then $p \prec q$ and $q$ is the best dominant. 
Lemma $1.3[14$, Corollary 3.4a, p.120] Let $q$ be analytic in $\mathbb{D}$, let $\phi$ be analytic in a domain D containing $q(\mathbb{D})$ and suppose

(1) $\operatorname{Re} \phi[q(z)]>0$ and either

(2) $q$ is convex, or

(3) $Q(z)=z q^{\prime}(z) \cdot \phi[q(z)]$ is starlike.

If $p$ is analytic in $\mathbb{D}$, with $p(0)=q(0), p(\mathbb{D}) \subset D$ and

$$
p(z)+z p^{\prime}(z) \phi[p(z)] \prec q(z),
$$

then $p(z) \prec q(z)$.

\section{Results associated with the lemniscate of Bernoulli}

In the first result, condition on $\beta$ is obtained so that the subordination

$$
1+\beta \frac{z p^{\prime}(z)}{p^{k}(z)} \prec \frac{1+A z}{1+B z} \quad(-1<B<A \leq 1)
$$

implies $p(z) \prec \sqrt{1+z}$.

Lemma 2.1 Let $|\beta| \geq 2^{(k+3) / 2}(A-B)+|B \beta|,-1<k \leq 3$. Let $p$ be an analyticfunction defined on $\mathbb{D}$ with $p(0)=1$ satisfying

$$
1+\beta \frac{z p^{\prime}(z)}{p^{k}(z)} \prec \frac{1+A z}{1+B z} \quad(-1<B<A \leq 1)
$$

then $p(z) \prec \sqrt{1+z}$.

Proof Let $q(z)=\sqrt{1+z}$. A computation shows that the function

$$
Q(z):=\beta \frac{z q^{\prime}(z)}{q^{k}(z)}=\frac{\beta z}{2(1+z)^{(k+1) / 2}} \quad(-1<k \leq 3)
$$

is starlike in the unit disk $\mathbb{D}$. Consider the subordination

$$
1+\beta \frac{z p^{\prime}(z)}{p^{k}(z)} \prec 1+\beta \frac{z q^{\prime}(z)}{q^{k}(z)}
$$

Thus in view of Lemma 1.1, it follows that $p(z) \prec q(z)$. In order to prove our result, we need to prove

$$
\frac{1+A z}{1+B z} \prec 1+\frac{\beta z q^{\prime}(z)}{q^{k}(z)}=1+\frac{\beta z}{2(1+z)^{(k+1) / 2}}:=h(z) .
$$

Let $w=\Phi(z)=\frac{1+A z}{1+B z}$. Then $\Phi^{-1}(w)=\frac{w-1}{A-B w}$. The subordination $\Phi(z) \prec h(z)$ is equivalent to $z \prec \Phi^{-1}(h(z))$. Thus in order to prove the result, we need only to show $\left|\Phi^{-1}\left(h\left(e^{i t}\right)\right)\right| \geq 1$. For $z=e^{i t},-\pi \leq t \leq \pi$, we have

$$
\left|\Phi^{-1}\left(h\left(e^{i t}\right)\right)\right| \geq \frac{|\beta|}{2(A-B)(2 \cos (t / 2))^{(k+1) / 2}+|B \beta|}=: g(t) .
$$


A calculation shows that $g(t)$ attains its minimum at $t=0$. Further, the value of $g(t)$ at $\pi$ or $-\pi$ comes out to be $1 /|B|$ which is naturally greater than the value at the extreme point $t=0$ because if $g(0) \geq g(\pi)$, then $(A-B)|\beta| \leq 0$ which is absurd. Thus

$$
g(0)=\frac{|\beta|}{2^{(k+3) / 2}(A-B)+|B \beta|} \geq 1
$$

for $|\beta| \geq 2^{(k+3) / 2}(A-B)+|B \beta|$. Hence $\Phi(z) \prec h(z)$, and the proof is complete now.

Next result depicts the condition on $\beta$ such that $1+\beta z p^{\prime}(z) \prec \sqrt{1+z}$ implies $p(z) \prec$ $(1+A z) /(1+B z)(-1 \leq B<A \leq 1)$. On subsequent lemmas, similar results are obtained by considering the expressions $1+\beta z p^{\prime}(z) / p(z)$ and $1+\beta z p^{\prime}(z) / p^{2}(z)$.

Lemma 2.2 Let $(A-B) \beta \geq \sqrt{2}(1+|B|)^{2}+(1-B)^{2}$ and $-1 \leq B<A \leq 1$. Let $p$ be an analytic function defined on $\mathbb{D}$ with $p(0)=1$ satisfying

$$
1+\beta z p^{\prime}(z) \prec \sqrt{1+z}
$$

then $p(z) \prec \frac{1+A z}{1+B z}$.

Proof Define the function $q: \mathbb{D} \rightarrow \mathbb{C}$ by

$$
q(z)=\frac{1+A z}{1+B z} \quad(-1 \leq B<A \leq 1)
$$

with $q(0)=1$. A computation shows that

$$
Q(z)=\beta z q^{\prime}(z)=\frac{\beta(A-B) z}{(1+B z)^{2}}
$$

and

$$
\frac{z Q^{\prime}(z)}{Q(z)}=\frac{1-B z}{1+B z} .
$$

Let $z=r e^{i t}, r \in(0,1),-\pi \leq t \leq \pi$. Then

$$
\begin{aligned}
\operatorname{Re}\left(\frac{1-B z}{1+B z}\right) & =\operatorname{Re}\left(\frac{1-B r e^{i t}}{1+B r e^{i t}}\right) \\
& =\frac{1-B^{2} r^{2}}{\left|1+B r e^{i t}\right|^{2}}
\end{aligned}
$$

Since $1-B^{2} r^{2}>0(|B| \leq 1,0<r<1)$ and so $\operatorname{Re}\left(z Q^{\prime}(z) / Q(z)\right)>0$, this shows that $Q$ is starlike in $\mathbb{D}$. It follows from Lemma 1.1 that the subordination

$$
1+\beta z p^{\prime}(z) \prec 1+\beta z q^{\prime}(z)
$$

implies $p(z) \prec q(z)$. Now we need to prove the following in order to prove the lemma:

$$
\sqrt{1+z} \prec 1+\beta z q^{\prime}(z)=1+\beta \frac{(A-B) z}{(1+B z)^{2}}=: h(z)
$$


Let $w=\Phi(z)=\sqrt{1+z}$. Then $\Phi^{-1}(w)=w^{2}-1$. The subordination $\Phi(z) \prec h(z)$ is equivalent to the subordination $z \prec \Phi^{-1}(h(z))$. Now in order to prove the result, it is enough to show $\left|\Phi^{-1}\left(h\left(e^{i t}\right)\right)\right| \geq 1, z=e^{i t},-\pi \leq t \leq \pi$. Now

$$
\left|\Phi^{-1}\left(h\left(e^{i t}\right)\right)\right|=\left|\left(1+\beta \frac{(A-B) e^{i t}}{\left(1+B e^{i t}\right)^{2}}\right)^{2}-1\right| \geq 1 \text { implies that }\left|1+\beta \frac{(A-B) e^{i t}}{\left(1+B e^{i t}\right)^{2}}\right| \geq \sqrt{2}
$$

Further,

$$
\begin{aligned}
\left|1+\beta \frac{(A-B) e^{i t}}{\left(1+B e^{i t}\right)^{2}}\right| & =\frac{\left|1+(2 B+\beta(A-B)) e^{i t}+B^{2} e^{2 i t}\right|}{\left|1+2 B e^{i t}+B^{2} e^{2 i t}\right|} \\
& \geq \frac{\operatorname{Re}\left(2 B+\beta(A-B)+B^{2} e^{i t}+e^{-i t}\right)}{1+2|B|+B^{2}} \\
& =\frac{2 B+\beta(A-B)+\left(1+B^{2}\right) \cos t}{(1+|B|)^{2}} \\
& \geq \frac{2 B+\beta(A-B)-\left(1+B^{2}\right)}{(1+|B|)^{2}} \geq \sqrt{2}
\end{aligned}
$$

for $(A-B) \beta \geq \sqrt{2}(1+|B|)^{2}+(1-B)^{2}$. Therefore $\Phi(z) \prec h(z)$ and this completes the proof.

Lemma 2.3 Let $(A-B) \beta \geq(\sqrt{2}-1)(1+|A|)(1+|B|)$ and $-1 \leq B<A \leq 1$. Let $p$ be an analytic function defined on $\mathbb{D}$ with $p(0)=1$ satisfying

$$
1+\beta \frac{z p^{\prime}(z)}{p(z)} \prec \sqrt{1+z},
$$

then $p(z) \prec \frac{1+A z}{1+B z}$.

Proof Let the function $q: \mathbb{D} \rightarrow \mathbb{C}$ be defined by

$$
q(z)=\frac{1+A z}{1+B z} \quad(-1 \leq B<A \leq 1) .
$$

A computation shows that

$$
Q(z):=\frac{\beta z q^{\prime}(z)}{q(z)}=\frac{\beta(A-B) z}{(1+A z)(1+B z)}
$$

and

$$
\frac{z Q^{\prime}(z)}{Q(z)}=\frac{1-A B z^{2}}{(1+A z)(1+B z)} .
$$

Let $z=r e^{i t}, r \in(0,1),-\pi \leq t \leq \pi$. Then

$$
\begin{aligned}
\operatorname{Re}\left(\frac{1-A B z^{2}}{(1+A z)(1+B z)}\right) & =\operatorname{Re}\left(\frac{1-A B r^{2} e^{2 i t}}{\left(1+A r e^{i t}\right)\left(1+B r e^{i t}\right)}\right) \\
& =\frac{\left(1-A B r^{2}\right)\left(1+(A+B) r \cos t+A B r^{2}\right)}{\left|1+A r e^{i t}\right|^{2}\left|1+B r e^{i t}\right|^{2}} .
\end{aligned}
$$


Since $1+A B r^{2}+(A+B) r \cos t \geq(1-A r)(1-B r)>0$ for $A+B \geq 0$ and, similarly, $1+A B r^{2}+$ $(A+B) r \cos t \geq(1+A r)(1+B r)>0$ for $A+B \leq 0$, it follows that $Q$ is starlike in $\mathbb{D}$. Lemma 1.1 suggests that the subordination

$$
1+\beta \frac{z p^{\prime}(z)}{p(z)} \prec 1+\beta \frac{z q^{\prime}(z)}{q(z)}
$$

implies $p(z) \prec q(z)$. Now we have to prove

$$
\sqrt{1+z} \prec 1+\beta \frac{z q^{\prime}(z)}{q(z)}=1+\frac{\beta(A-B) z}{(1+A z)(1+B z)}=: h(z) .
$$

Let $w=\Phi(z)=\sqrt{1+z}$. Then $\Phi^{-1}(w)=w^{2}-1$. The subordination $\Phi(z) \prec h(z)$ is equivalent to the subordination $z \prec \Phi^{-1}(h(z))$. Now in order to prove the result, it is enough to show $\left|\Phi^{-1}\left(h\left(e^{i t}\right)\right)\right| \geq 1,-\pi \leq t \leq \pi$. Now

$$
\begin{aligned}
& \left|\Phi^{-1}\left(h\left(e^{i t}\right)\right)\right|=\left|\left(1+\frac{\beta(A-B) e^{i t}}{\left(1+A e^{i t}\right)\left(1+B e^{i t}\right)}\right)^{2}-1\right| \geq 1 \quad \text { implies that } \\
& \left|1+\frac{\beta(A-B) e^{i t}}{\left(1+A e^{i t}\right)\left(1+B e^{i t}\right)}\right| \geq \sqrt{2} .
\end{aligned}
$$

Further,

$$
\begin{aligned}
\left|1+\frac{\beta(A-B) e^{i t}}{\left(1+A e^{i t}\right)\left(1+B e^{i t}\right)}\right| & \geq \operatorname{Re}\left(1+\frac{\beta(A-B) e^{i t}}{\left(1+A e^{i t}\right)\left(1+B e^{i t}\right)}\right) \\
& \geq 1+\frac{(A-B) \beta}{(1+|A|)(1+|B|)} \geq \sqrt{2}
\end{aligned}
$$

for $(A-B) \beta \geq(\sqrt{2}-1)(1+|A|)(1+|B|)$. Therefore $\Phi(z) \prec h(z)$ and this completes the proof.

Lemma 2.4 Let $(A-B) \beta \geq(\sqrt{2}-1)(1+|A|)^{2}+(1-A)^{2}$ and $-1 \leq B<A \leq 1$. Let $p$ be an analytic function defined on $\mathbb{D}$ with $p(0)=1$ satisfying

$$
1+\beta \frac{z p^{\prime}(z)}{p^{2}(z)} \prec \sqrt{1+z},
$$

then $p(z) \prec \frac{1+A z}{1+B z}$.

Proof Let the function $q: \mathbb{D} \rightarrow \mathbb{C}$ be defined by

$$
q(z)=\frac{1+A z}{1+B z} \quad(-1 \leq B<A \leq 1)
$$

with $q(0)=1$. Then

$$
Q(z)=\frac{\beta z q^{\prime}(z)}{q^{2}(z)}=\frac{\beta(A-B) z}{(1+A z)^{2}}
$$


and

$$
\frac{z Q^{\prime}(z)}{Q(z)}=\frac{1-A z}{1+A z}
$$

Let $z=r e^{i t},-\pi \leq t \leq \pi, 0<r<1$. Then

$$
\operatorname{Re}\left(\frac{1-A z}{1+A z}\right)=\frac{1-A^{2} r^{2}}{\left|1+A r e^{i t}\right|^{2}}
$$

Since $1-A^{2} r^{2}>0(|A| \leq 1,0<r<1)$. Hence $\operatorname{Re}\left(z Q^{\prime}(z)\right) / Q(z)>0$, this shows that $Q$ is starlike in $\mathbb{D}$. An application of Lemma 1.1 reveals that the subordination

$$
1+\beta \frac{z p^{\prime}(z)}{p^{2}(z)} \prec 1+\beta \frac{z q^{\prime}(z)}{q^{2}(z)}
$$

implies $p(z) \prec q(z)$. Now our result is established if we prove

$$
\sqrt{1+z} \prec 1+\beta \frac{z q^{\prime}(z)}{q^{2}(z)}=1+\beta \frac{(A-B) z}{(1+A z)^{2}}=: h(z) .
$$

The rest of the proof is similar to that of Lemma 2.2, and therefore it is skipped here.

In the next result, the condition on $\beta$ is obtained so that $p(z)+\beta z p^{\prime}(z) \prec \sqrt{1+z}$ implies $p(z) \prec \sqrt{1+z}$. On subsequent lemmas, similar results are discussed by considering the expressions $p(z)+\beta z p^{\prime}(z) / p(z)$ and $p(z)+\beta z p^{\prime}(z) / p^{2}(z)$.

Lemma 2.5 Let $p$ be an analytic function defined on $\mathbb{D}$ with $p(0)=1$ satisfying $p(z)+$ $\beta z p^{\prime}(z) \prec \sqrt{1+z}, \beta>0$. Then $p(z) \prec \sqrt{1+z}$.

Proof Define the function $q: \mathbb{D} \rightarrow \mathbb{C}$ by $q(z)=\sqrt{1+z}$ with $q(0)=1$. Since $q(\mathbb{D})=\left\{w: \mid w^{2}-\right.$ $1 \mid<1\}$ is the right half of the lemniscate of Bernoulli, $q(\mathbb{D})$ is a convex set, and hence $q$ is a convex function. Let us define $\phi(w)=\beta$, then

$$
\operatorname{Re} \phi[q(z)]=\beta>0 .
$$

Consider the function $Q$ defined by

$$
Q(z):=z q^{\prime}(z) \phi(q(z))=\beta \frac{z}{2 \sqrt{1+z}} .
$$

Further,

$$
\begin{aligned}
\operatorname{Re}\left(\frac{z Q^{\prime}(z)}{Q(z)}\right) & =1-\operatorname{Re}\left(\frac{z}{2(1+z)}\right) \\
& \geq \frac{3}{4}>0 .
\end{aligned}
$$

Thus the function $Q$ is starlike, and the result now follows by an application of Lemma 1.3. 
Lemma 2.6 Let $p$ be an analytic function defined on $\mathbb{D}$ with $p(0)=1$ satisfying

$$
p(z)+\beta \frac{z p^{\prime}(z)}{p(z)} \prec \sqrt{1+z}, \quad \beta>0 .
$$

Then $p(z) \prec \sqrt{1+z}$.

Proof As before, let $q$ be given by $q(z)=\sqrt{1+z}$ with $q(0)=1$. Then $q$ is a convex function. Let us define $\phi(w)=\beta / w$. Since $q(\mathbb{D})=\left\{w:\left|w^{2}-1\right|<1\right\}$ is the right half of the lemniscate of Bernoulli, so

$$
\operatorname{Re} \phi[q(z)]=\frac{\beta}{|\sqrt{1+z}|^{2}} \operatorname{Re}(\sqrt{1+z})>0 .
$$

Consider the function $Q$ defined by

$$
Q(z):=\beta \frac{z q^{\prime}(z)}{q(z)}=\beta \frac{z}{2(1+z)} .
$$

Further,

$$
\operatorname{Re}\left(\frac{z Q^{\prime}(z)}{Q(z)}\right)=1-\operatorname{Re}\left(\frac{z}{1+z}\right) \geq \frac{1}{2}>0 .
$$

Thus the function $Q$ is starlike, and the result now follows by an application of Lemma 1.3.

Lemma 2.7 Let $p$ be an analytic function defined on $\mathbb{D}$ with $p(0)=1$ satisfying

$$
p(z)+\beta \frac{z p^{\prime}(z)}{p^{2}(z)} \prec \sqrt{1+z}, \quad \beta>0 .
$$

Then $p(z) \prec \sqrt{1+z}$.

Proof Let $q$ be given by $q(z)=\sqrt{1+z}$ with $q(0)=1$. Then $q$ is a convex function. Let us define $\phi(w)=\beta / w^{2}$ and

$$
\operatorname{Re} \phi[q(z)]=\operatorname{Re}\left(\frac{\beta}{1+z}\right)>\frac{\beta}{2}>0 .
$$

Consider the function $Q$ defined by

$$
Q(z):=\beta \frac{z q^{\prime}(z)}{q^{2}(z)}=\beta \frac{z}{2(1+z)^{\frac{3}{2}}} .
$$

Further,

$$
\operatorname{Re}\left(\frac{z Q^{\prime}(z)}{Q(z)}\right)=1-\frac{3}{2} \operatorname{Re}\left(\frac{z}{1+z}\right) \geq \frac{1}{4}>0 .
$$

Thus the function $Q$ is starlike, and the result now follows by an application of Lemma 1.3. 
In the next result, the condition on $\beta$ is obtained such that $p(z)+\beta z p^{\prime}(z) / p(z) \prec \sqrt{1+z}$ implies that $p(z) \prec(1+A z) /(1+B z)$.

Lemma 2.8 Let $-1 \leq B<A \leq 1,(A-B) \beta \geq \sqrt{2}(1+|A|)(1+|B|)+|A|^{2}-1$ and

$$
\frac{1}{\beta} \geq \max \left\{0, \frac{A-B}{(1+|A|)(1+|B|)}-\frac{1-|B|}{1+|B|}\right\} .
$$

Let $p$ be an analytic function defined on $\mathbb{D}$ with $p(0)=1$ satisfying

$$
p(z)+\beta \frac{z p^{\prime}(z)}{p(z)} \prec \sqrt{1+z} .
$$

Then $p(z) \prec \frac{1+A z}{1+B z}$.

Proof Define the function $q: \mathbb{D} \rightarrow \mathbb{C}$ by $q(z)=(1+A z) /(1+B z),-1 \leq B<A \leq 1$. Consider the subordination

$$
p(z)+\beta \frac{z p^{\prime}(z)}{p(z)} \prec q(z)+\beta \frac{z q^{\prime}(z)}{q(z)} .
$$

Thus, in view of Lemma 1.2, the above subordination can be written as (1.1) by defining the functions $v$ and $\varphi$ as $v(w):=w$ and $\varphi(w):=\beta / w(\beta \neq 0)$. Clearly, the functions $v$ and $\varphi$ are analytic in $\mathbb{C}$ and $\varphi(w) \neq 0$. Let the functions $Q(z)$ and $h(z)$ be defined by

$$
Q(z):=z q^{\prime}(z) \varphi(q(z))=\beta \frac{z q^{\prime}(z)}{q(z)}
$$

and

$$
h(z):=v(q(z))+Q(z)=q(z)+\beta \frac{z q^{\prime}(z)}{q(z)} .
$$

A computation shows that $Q(z)$ is starlike univalent in $\mathbb{D}$. Further,

$$
\frac{z h^{\prime}(z)}{Q(z)}=\frac{1}{\beta}+1+\frac{z q^{\prime \prime}(z)}{q^{\prime}(z)}-\frac{z q^{\prime}(z)}{q(z)} .
$$

Let $z=e^{i t},-\pi \leq t \leq \pi$. Then

$$
\begin{aligned}
\operatorname{Re}\left(\frac{e^{i t} h^{\prime}\left(e^{i t}\right)}{Q\left(e^{i t}\right)}\right) & =\frac{1}{\beta}+\operatorname{Re}\left(\frac{1-B e^{i t}}{1+B e^{i t}}-\frac{(A-B) e^{i t}}{\left(1+A e^{i t}\right)\left(1+B e^{i t}\right)}\right) \\
& \geq \frac{1}{\beta}+\frac{1-|B|}{1+|B|}-\frac{A-B}{(1+|A|)(1+|B|)}>0 .
\end{aligned}
$$

Thus by Lemma 1.2, it follows that $p(z) \prec q(z)$. In order to prove our result, we need to prove that

$$
\Phi(z):=\sqrt{1+z} \prec q(z)+\beta \frac{z q^{\prime}(z)}{q(z)}=\frac{1+A z}{1+B z}+\frac{\beta(A-B) z}{(1+A z)(1+B z)}:=h(z) .
$$


The subordination $\Phi(z) \prec h(z)$ is equivalent to the subordination $z \prec \Phi^{-1}(h(z))$. Now in order to prove the result, it is enough to show $\left|\Phi^{-1}\left(h\left(e^{i t}\right)\right)\right| \geq 1,-\pi \leq t \leq \pi$. Now

$$
\left|\Phi^{-1}\left(h\left(e^{i t}\right)\right)\right|=\left|\left(\frac{1+A e^{i t}}{1+B e^{i t}}+\frac{\beta(A-B) e^{i t}}{\left(1+A e^{i t}\right)\left(1+B e^{i t}\right)}\right)^{2}-1\right| \geq 1
$$

implies

$$
\left|\frac{1+A e^{i t}}{1+B e^{i t}}+\frac{\beta(A-B) e^{i t}}{\left(1+A e^{i t}\right)\left(1+B e^{i t}\right)}\right| \geq \sqrt{2} .
$$

Further,

$$
\begin{aligned}
\left|\frac{1+A e^{i t}}{1+B e^{i t}}+\frac{\beta(A-B) e^{i t}}{\left(1+A e^{i t}\right)\left(1+B e^{i t}\right)}\right| & \geq \operatorname{Re}\left(\frac{1+A e^{i t}}{1+B e^{i t}}+\frac{\beta(A-B) e^{i t}}{\left(1+A e^{i t}\right)\left(1+B e^{i t}\right)}\right) \\
& \geq \frac{1-|A|}{1+|B|}+\frac{(A-B) \beta}{(1+|A|)(1+|B|)} \geq \sqrt{2}
\end{aligned}
$$

for $(A-B) \beta \geq \sqrt{2}(1+|A|)(1+|B|)+|A|^{2}-1$. This completes the proof.

\section{Sufficient condition for Janowski starlikeness}

The following first two results (Lemmas 3.1,3.2) are essentially due to Ali et al. [13, Lemmas 2.1, 2.10]. However, an alternate proof of the same result, which is much easier than that given by Ali et al. [13], is presented below.

Lemma 3.1 Assume that $-1 \leq B<A \leq 1,-1 \leq E<D \leq 1$ and $\beta(A-B) \geq(D-E)\left(1+B^{2}\right)+$ $|2 B(D-E)-E \beta(A-B)|$. Let $p$ be an analytic function defined on $\mathbb{D}$ with $p(0)=1$ satisfying

$$
1+\beta z p^{\prime}(z) \prec \frac{1+D z}{1+E z}, \quad \beta \neq 0 .
$$

Then $p(z) \prec \frac{1+A z}{1+B z}$.

Proof Define the function $q: \mathbb{D} \rightarrow \mathbb{C}$ by

$$
q(z)=\frac{1+A z}{1+B z}, \quad-1 \leq B<A \leq 1 .
$$

Then $q$ is convex in $\mathbb{D}$ with $q(0)=1$. Further computation shows that

$$
Q(z)=\beta z q^{\prime}(z)=\frac{\beta(A-B) z}{(1+B z)^{2}}
$$

and $Q$ is starlike in $\mathbb{D}$. It follows from Lemma 1.1 that the subordination

$$
1+\beta z p^{\prime}(z) \prec 1+\beta z q^{\prime}(z)
$$

implies $p(z) \prec q(z)$. In view of the above result, it is sufficient to prove

$$
\frac{1+D z}{1+E z} \prec 1+\beta z q^{\prime}(z)=1+\beta \frac{(A-B) z}{(1+B z)^{2}}=h(z) .
$$


Let $w=\Phi(z)=\frac{1+D z}{1+E z}$. Then $\Phi^{-1}(w)=\frac{w-1}{D-E w}$ and

$$
\begin{aligned}
\Phi^{-1}(h(z)) & =\frac{\beta(A-B) z}{D(1+B z)^{2}-E(1+B z)^{2}-\beta E(A-B) z} \\
& =\frac{\beta(A-B) z}{(D-E)\left(1+B^{2} z^{2}\right)+(2 B(D-E)-\beta E(A-B)) z} .
\end{aligned}
$$

Let $z=e^{i t}, \pi \leq t \leq \pi$. Thus

$$
\left|\Phi^{-1}\left(h\left(e^{i t}\right)\right)\right| \geq \frac{|\beta|(A-B)}{(D-E)\left(1+B^{2}\right)+|(2 B(D-E)-\beta E(A-B))|} \geq 1
$$

for $|\beta|(A-B) \geq(D-E)\left(1+B^{2}\right)+|(2 B(D-E)-E \beta(A-B))|$. Hence $q(\mathbb{D}) \subset h(\mathbb{D})$, that is, $q(z) \prec h(z)$, this completes the proof.

It should be noted that Ali et al. [13] made the assumption $A B>0$ in order to prove the result [13, Lemma 2.10], whereas in the following lemma this condition has been dropped.

Lemma 3.2 Assume that $-1 \leq B<A \leq 1,-1 \leq E<D \leq 1$ and $\beta(A-B) \geq(D-E)(1+$ $|A B|)+|(A+B)(D-E)-E \beta(A-B)|$. Let $p$ be an analytic function defined on $\mathbb{D}$ with $p(0)=1$ satisfying

$$
1+\beta \frac{z p^{\prime}(z)}{p(z)} \prec \frac{1+D z}{1+E z}, \quad \beta \neq 0 .
$$

Then $p(z) \prec \frac{1+A z}{1+B z}$.

Proof As above, define the function $q: \mathbb{D} \rightarrow \mathbb{C}$ by

$$
q(z)=\frac{1+A z}{1+B z}, \quad-1 \leq B<A \leq 1 .
$$

Then $q$ is convex in $\mathbb{D}$ with $q(0)=1$. A computation shows that

$$
Q(z)=\frac{\beta z q^{\prime}(z)}{q(z)}=\frac{\beta(A-B) z}{(1+A z)(1+B z)}
$$

and $Q$ is starlike in $\mathbb{D}$. It follows from Lemma 1.1 that the subordination

$$
1+\beta \frac{z p^{\prime}(z)}{p(z)} \prec 1+\beta \frac{z q^{\prime}(z)}{q(z)}
$$

implies $p(z) \prec q(z)$. Now we need to prove

$$
\frac{1+D z}{1+E z} \prec 1+\beta \frac{z q^{\prime}(z)}{q(z)}=1+\beta \frac{(A-B) z}{(1+B z)^{2}}=h(z) .
$$

Let $w=\Phi(z)=\frac{1+D z}{1+E z}$. Then $\Phi^{-1}(w)=\frac{w-1}{D-E w}$ and

$$
\begin{aligned}
\Phi^{-1}(h(z)) & =\frac{\beta(A-B) z}{(D-E)(1+A z)(1+B z)-\beta E(A-B) z} \\
& =\frac{\beta(A-B) z}{(D-E)\left(1+A B z^{2}\right)+((A+B)(D-E)-\beta E(A-B)) z} .
\end{aligned}
$$


Let $z=e^{i t}, \pi \leq t \leq \pi$. Thus

$$
\left|\Phi^{-1}\left(h\left(e^{i t}\right)\right)\right| \geq \frac{|\beta|(A-B)}{(D-E)(1+|A B|)+|(A+B)(D-E)-\beta E(A-B)|} \geq 1
$$

for $|\beta|(A-B) \geq(D-E)(1+|A B|)+|(A+B)(D-E)-E \beta(A-B)|$. Hence $q(\mathbb{D}) \subset h(\mathbb{D})$, that is, $q(z) \prec h(z)$, this completes the proof.

Lemma 3.3 Assume that $-1 \leq B<A \leq 1,-1 \leq E<D \leq 1$ and $|\beta|(A-B) \geq(D-E)\left(1+A^{2}\right)+$ $|2 A(D-E)-E \beta(A-B)|$. Let $p$ be an analytic function defined on $\mathbb{D}$ with $p(0)=1$ satisfying

$$
1+\beta \frac{z p^{\prime}(z)}{p^{2}(z)} \prec \frac{1+D z}{1+E z} .
$$

Then $p(z) \prec \frac{1+A z}{1+B z}$.

Proof Define the function $q: \mathbb{D} \rightarrow \mathbb{C}$ by

$$
q(z)=\frac{1+A z}{1+B z}, \quad-1 \leq B<A \leq 1
$$

Then $q$ is convex in $\mathbb{D}$ with $q(0)=1$. A computation shows that

$$
Q(z)=\frac{\beta z q^{\prime}(z)}{q^{2}(z)}=\frac{\beta(A-B) z}{(1+A z)^{2}}
$$

and

$$
\frac{z Q^{\prime}(z)}{Q(z)}=\frac{1-A z}{1+A z}
$$

As before, a computation shows $Q$ is starlike in $\mathbb{D}$. It follows from Lemma 1.1 that the subordination

$$
1+\beta \frac{z p^{\prime}(z)}{p^{2}(z)} \prec 1+\beta \frac{z q^{\prime}(z)}{q^{2}(z)}
$$

implies $p(z) \prec q(z)$. To prove result, it is enough to show that

$$
\frac{1+D z}{1+E z} \prec 1+\beta \frac{z q^{\prime}(z)}{q^{2}(z)}=1+\beta \frac{(A-B) z}{(1+A z)^{2}}=h(z) .
$$

The remaining part of the proof is similar to that of Lemma 3.1, and therefore it is skipped here.

Remark 3.4 When $\beta=1$, Lemma 3.3 reduces to [13, Lemma 2.6] due to Ali et al. 


\section{Author details}

${ }^{1}$ Department of Applied Mathematics, Delhi Technological University, Delhi, 110042, India. ${ }^{2}$ Department of Mathematics, University of Delhi, Delhi, 110007, India. ${ }^{3}$ Department of Applied Mathematics, Pukyong National University, Busan, 608-737, South Korea.

\section{Acknowledgements}

Dedicated to Professor Hari M Srivastava.

The research work is supported by a grant from University of Delhi and also by the Basic Science Research Program through the National Research Foundation of Korea (NRF) funded by the Ministry of Education, Science and Technology (No. 2012-0002619).

Received: 10 January 2013 Accepted: 15 March 2013 Published: 16 April 2013

\section{References}

1. Janowski, W: Extremal problems for a family of functions with positive real part and for some related families. Ann. Pol. Math. 23, 159-177 (1970/1971)

2. Polatoğlu, Y, Bolcal, M: Some radius problem for certain families of analytic functions. Turk. J. Math. 24(4), 401-412 (2000)

3. Sokół, J, Stankiewicz, J: Radius of convexity of some subclasses of strongly starlike functions. Zesz. Nauk. Politech. Rzesz. Mat. 19, 101-105 (1996)

4. Ali, RM, Cho, NE, Ravichandran, V, Kumar, SS: First order differential subordinations for functions associated with the lemniscate of Bernoulli. Taiwan. J. Math. 16(3), 1017-1026 (2012)

5. Ali, RM, Jain, NK, Ravichandran, V: Radii of starlikeness associated with the lemniscate of Bernoulli and the left-half plane. Appl. Math. Comput. 218(11), 6557-6565 (2012)

6. Paprocki, E, Sokół, J: The extremal problems in some subclass of strongly starlike functions. Zesz. Nauk. Politech. Rzesz. Mat. 20, 89-94 (1996)

7. Ali, RM, Cho, NE, Jain, NK, Ravichandran, V: Radii of starlikeness and convexity of functions defined by subordination with fixed second coefficient. Filomat 26(3), 553-561 (2012)

8. Sokół, J: Radius problems in the class $\mathcal{S} \mathcal{L}^{*}$. Appl. Math. Comput. 214(2), 569-573 (2009)

9. Silverman, H: Convex and starlike criteria. Int. J. Math. Math. Sci. 22(1), 75-79 (1999)

10. Obradowič, M, Tuneski, N: On the starlike criteria defined by Silverman. Zesz. Nauk. Politech. Rzesz. Mat. 24, 59-64 (2001)

11. Tuneski, N: On the quotient of the representations of convexity and starlikeness. Math. Nachr. 248/249,200-203 (2003)

12. Nunokawa, M, Owa, S, Saitoh, H, Ahuja, OP: On the quotient of the analytic representations of convex and starlike functions. Sūrikaisekikenkyūsho Kōkyūroku 1112, 63-69 (1999)

13. Ali, RM, Ravichandran, V, Seenivasagan, N: Sufficient conditions for Janowski starlikeness. Int. J. Math. Math. Sci. 2007, Article ID 62925 (2007)

14. Miller, SS, Mocanu, PT: Differential Subordinations: Theory and Applications. Pure and Applied Mathematics, vol. 225. Dekker, New York (2000)

doi:10.1186/1029-242X-2013-176

Cite this article as: Kumar et al.: Sufficient conditions for starlike functions associated with the lemniscate of

Bernoulli. Journal of Inequalities and Applications 2013 2013:176.

\section{Submit your manuscript to a SpringerOpen ${ }^{\odot}$ journal and benefit from:}

- Convenient online submission

- Rigorous peer review

Immediate publication on acceptance

- Open access: articles freely available online

- High visibility within the field

- Retaining the copyright to your article 